\title{
Alcohol Consumption Is a Risk Factor for Lower Extremity Arterial Disease in Chinese Patients with T2DM
}

\author{
Shanshan Yang, ${ }^{1,2}$ Shuang Wang, ${ }^{3}$ Bo Yang, ${ }^{3}$ Jinliang Zheng, ${ }^{3}$ \\ Yuping Cai, ${ }^{3}$ and Zhengguo Yang ${ }^{3}$ \\ ${ }^{1}$ Institute of Geriatrics, Beijing Key Laboratory of Ageing and Geriatrics, State Key Laboratory of Kidney Disease, \\ Chinese PLA General Hospital, 28 Fuxing Road, Beijing 100853, China \\ ${ }^{2}$ Jinan Military Area CDC, Jinan, Shandong 250014, China \\ ${ }^{3}$ Department of Nephrology and Endocrinology, PLA 148th Hospital, Zibo 255300, China
}

Correspondence should be addressed to Zhengguo Yang; yangzhanq@163.com

Received 7 March 2017; Revised 22 May 2017; Accepted 28 May 2017; Published 6 July 2017

Academic Editor: Nikolaos Papanas

Copyright $\odot 2017$ Shanshan Yang et al. This is an open access article distributed under the Creative Commons Attribution License, which permits unrestricted use, distribution, and reproduction in any medium, provided the original work is properly cited.

Objective. To investigate the relationship between alcohol consumption and diabetic lower extremity arterial disease (LEAD) in hospitalized patients with type 2 diabetes mellitus (T2DM). Methods. We evaluated 138 hospitalized patients with T2DM who consumed alcohol and 833 who did not. We used propensity score matching to reduce the confounding bias between groups. Additionally, a logistic regression analysis was performed with the matched data to evaluate the LEAD risk. Results. In total, 119 pairs of patients who did and did not consume alcohol were matched. According to the logistic regression analysis, patients who consumed $>8 \mathrm{U}$ of alcohol/day had a higher risk of LEAD (odds ratio (OR): 6.35, 95\% confidence interval (CI): 1.78-22.65) than patients who did not consume alcohol. Additionally, after adjusting for age, gender, region, occupation, smoking status, body mass index, weight change, and duration of diabetes, the OR of peripheral artery disease after $>20$ years of alcohol consumption was 3.48 (95\% CI: 1.09-11.15). Furthermore, we observed a significant dose-response relationship between alcohol consumption and LEAD. Conclusions. Alcohol consumption may be a risk factor of LEAD in patients with T2DM. Patients with T2DM should be advised to stop drinking, to prevent the onset of LEAD.

\section{Introduction}

Lower extremity arterial disease (LEAD) is one of the most common complications of diabetes and harms the peripheral arteries via multiple pathways [1]. LEAD is also associated with healing failure, amputation, cardiovascular events, and an increased risk of premature mortality [2,3]. Furthermore, LEAD is a pathological process, and only one in four LEAD patients survives for more than 10 years. Approximately 10 million men and women in the United States suffer from LEAD [4], and in Chinese patients with diabetes older than 50 years, the prevalence of LEAD ranges from 16.9 to $23.8 \%$ [5]. Compared with individuals without diabetes mellitus (DM), patients with DM have a 2-fold higher risk of LEAD and of having an earlier onset age, a more serious illness, more lesions, and a worse prognosis [6]. The rate at which patients with LEAD eventually require an amputation is as high as 33\% [7]. In 1997, the medical cost of a diabetic foot ulcer in the United States was $\$ 10,831$ and the average hospital stay length was 8.9 days; the medical cost of an amputation was $\$ 17,302$ and the hospital stay length was 12 days, far exceeding the lengths of stay for coronary bypass surgery (9.9 days) and myocardial infarction (6.9 days) [8]. In China, the average duration of hospitalization for patients with LEAD undergoing amputation was 26 days and the average cost was 14,906 yuan [5].

Considering the serious health hazards of LEAD, a comprehensive identification of preventable risk factors of LEAD is important to improving patients' quality of life and reducing the associated medical costs. Smoking, ageing, race/ethnicity, increased levels of inflammatory markers, homocysteinaemia, and abdominal obesity are currently identified as risk factors 
for LEAD [9]. However, the association between alcohol use and LEAD remains unclear. Alcohol consumers with peripheral artery disease (PAD) were reported to have a lower mortality than patients with PAD who did not consume alcohol. On the other hand, heavy drinking has been reported to be a risk factor for PAD, whereas other studies have not identified an association between alcohol use and LEAD [9-12]. In addition, in these studies, the measures of alcohol consumption were not consistent, and the demographic characteristics of the groups who did and did not consume alcohol differed significantly. Studies on this topic in the Chinese population are limited. Thus, we designed a study to assess the association between alcohol consumption and LEAD in Chinese patients with type 2 diabetes mellitus (T2DM) using U (an international measure of ethanol) to measure alcohol consumption and propensity score matching (PSM) to control for differences in characteristics between patients who did and did not consume alcohol.

\section{Design and Methods}

2.1. Study Sample. We used clinical data from the Department of Nephrology and Endocrinology, PLA 148th Hospital. Of the 1025 inpatients (from January 2010 to December 2012), we excluded 25 inpatients with type 1 DM and 11 adult inpatients with latent autoimmune diabetes, and we recruited 989 (507 men and 482 women) as our participants.

We collected data regarding each participant's gender, age, occupation, region of residence, alcohol and smoking habits, T2DM duration, and LEAD status.

2.2. Measurements. T2DM was defined according to the American Diabetes Association criteria [13]. LEAD was diagnosed as follows: (1) an ankle-brachial index $(\mathrm{ABI})<0.90$, which is the systolic blood pressure at the ankle divided by the systolic blood pressure of the arm; (2) an ABI $>1.3$ and a toe-brachial index (TBI) $<0.7$; and (3) intermittent claudication, $0.9<\mathrm{ABI}<1.3$, and an $\mathrm{ABI}$ that is reduced by $15-20 \%$ after an exercise tolerance test [14]. The ABI was measured in all subjects, and the TBI was measured in subjects with an ABI > 1.3 using a Vista AVS system (Summit Doppler Systems Inc., Golden, CO 80403, USA).

An alcohol user was defined as a regular drinker who consumed alcohol almost every day and had regularly consumed alcohol for more than half a year. This definition was used because the average alcohol consumption of occasional drinkers is difficult to determine [15]. The participants' alcohol use status was defined by asking the question "Are you a regular drinker who has used alcohol almost every day for more than a half year?" The duration and quantity of alcohol consumption were defined by asking the questions "How many years have you been a regular drinker?" and "Do you drink more than two standard glasses (approximately $250 \mathrm{ml}$ ) of white spirits or 2.5 bottles $/ 5$ cans (approximately $1,500 \mathrm{ml}$ ) of beer a day on average?" The answers to these questions were either "A. Yes" or "B. No." A volume of $1 \mathrm{U}$ of consumed alcohol equals $10 \mathrm{ml}$ or $8 \mathrm{~g}$ of ethanol, which corresponds to the amount of alcohol a normal $(60 \mathrm{~kg})$ adult can metabolize in 1 hour [16]. Thus, we defined a heavy drinker as someone who consumed $8 \mathrm{U}$ of alcohol a day on average, that is, a person who metabolized alcohol at least 8 hours every day.

This information was collected by a primary nurse. The patients' answers to the questions on alcohol use were confirmed by the patients and their relatives to ensure the accuracy of the information. Central obesity was defined as a waist circumference (WC) $>90 \mathrm{~cm}$ in men and $>80 \mathrm{~cm}$ in women [17].

2.3. Statistical Analysis. SPSS version 19.0 was used to analyse the data. The significance level for all tests was set at a two-tailed $\alpha$ value of 0.05 . The differences in means and proportions were evaluated using Student's $t$-test and the chi-square test, respectively. Logistic regression models were used to identify the risk of alcohol use.

PSM [18] was used to match the groups of those who did and did not consume alcohol. Gender, age, region, occupation, smoking status, body mass index (BMI), WC, and the duration of T2DM were included as covariates. We used the nearestneighbour matching to match former smokers with current smokers at a $1: 1$ ratio with a calliper width of 0.02 [19].

2.4. Ethical Considerations. The Committee for Medical Ethics of the Chinese PLA General Hospital examined and approved our study. Before completing the questionnaire, each involved participant signed an informed consent form.

\section{Results}

Nine hundred and eighty-nine (507 men and 482 women) inpatients were involved in our study before PSM. The average age was $56.8 \pm 11.6$ years (range: $14-93$ years). The average ages of patients who did and did not consume alcohol were $52.3 \pm 11.3$ years (range: $28-85$ years) and 57.5 \pm 11.3 years (range: $14-93$ years), respectively. The general characteristics (age, gender, origin, occupation, smoking status, BMI, and central obesity) of the participants are shown in Table 1. Compared with the group of alcohol consumers, the group of patients who did not consume alcohol comprised more women, more workers engaged in hard physical labour, fewer smokers, and patients who were older and had more central obesity and longer durations of T2DM (5.4 \pm 6.0 years versus $7.5 \pm 6.6$ years; $P<0.05$ ).

After PSM, 238 participant pairs were matched, and the two groups were balanced for age, gender, occupation, smoking status, BMI, central obesity, and T2DM duration (with and without alcohol consumption: $5.8 \pm 6.3$ years versus 5.9 \pm 6.1 years, resp.; $P=0.937$ ) (Table 1 ).

According to the logistic regression analysis, patients who consumed alcohol had a higher risk of LEAD (OR: 2.75, 95\% CI: 1.11-6.80) than patients who did not after adjusting for age, gender, region, occupation, smoking status, BMI, WC, and T2DM duration. Regarding the risk of LEAD after adjusting for alcohol use $\leq 8 \mathrm{U} /$ day and $>8 \mathrm{U} /$ day, the odds ratios (ORs) were 2.07 (95\% confidence interval (CI): 0.78-5.54, $P>0.05$ ) and 6.35 (95\% CI: 1.78-22.65, $P<0.05)$, respectively. We also observed a dose-response relationship between the units of alcohol consumed per 
TABLe 1: Demographic characteristics of the participants according to alcohol use before and after PSM.

\begin{tabular}{|c|c|c|c|c|c|c|c|}
\hline \multirow{2}{*}{ Group } & \multirow{2}{*}{$\begin{array}{l}\text { Number }(\%) \\
\text { Total } n=971\end{array}$} & \multicolumn{3}{|c|}{ Alcohol use (before PSM) } & \multicolumn{3}{|c|}{ Alcohol use (after PSM) } \\
\hline & & Yes $(n=138)$ & No $(n=833)$ & $P$ & Yes $(n=119)$ & No $(n=119)$ & $P$ \\
\hline Age (years) & & & & $<0.001$ & & & 0.534 \\
\hline$\leq 40$ & $81(8.3)$ & $22(15.9)$ & $59(7.1)$ & & $16(13.4)$ & $15(12.6)$ & \\
\hline $60-69$ & $529(54.5)$ & $79(57.2)$ & $450(54.0)$ & & $68(57.1)$ & $76(63.9)$ & \\
\hline$\geq 70$ & $361(37.2)$ & $37(26.8)$ & $324(38.9)$ & & $35(29.4)$ & $28(23.5)$ & \\
\hline Gender & & & & $<0.001$ & & & 1.000 \\
\hline Male & $498(51.3)$ & $136(98.6)$ & $362(43.5)$ & & $117(98.3)$ & $117(98.3)$ & \\
\hline Female & $473(48.7)$ & $2(1.4)$ & $471(56.5)$ & & $2(1.7)$ & $2(1.7)$ & \\
\hline Occupation & & & & 0.01 & & & 0.562 \\
\hline White collar & $103(10.6)$ & $22(15.9)$ & $81(9.7)$ & & $84(70.6)$ & $77(64.7)$ & \\
\hline Light physical labourer & $117(12.0)$ & $23(16.7)$ & $94(11.3)$ & & $19(16.0)$ & $25(21.0)$ & \\
\hline Hard physical labourer & $751(77.3)$ & $93(67.4)$ & $658(79.0)$ & & $16(13.4)$ & $17(14.3)$ & \\
\hline Region & & & & 0.756 & & & 0.408 \\
\hline Shandong province & $940(96.8)$ & $133(96.4)$ & 807 (96.9) & & $117(98.3)$ & $115(96.6)$ & \\
\hline Other province & $31(3.2)$ & $5(3.6)$ & $26(3.1)$ & & $2(1.7)$ & $4(3.4)$ & \\
\hline Smoker & & & & $<0.001$ & & & 0.512 \\
\hline Yes & $182(18.7)$ & $90(65.2)$ & $92(11.0)$ & & $71(59.7)$ & $66(55.5)$ & \\
\hline No & $789(81.3)$ & $48(34.8)$ & $741(89.0)$ & & $48(40.3)$ & $53(44.5)$ & \\
\hline BMI & & & & 0.038 & & & 0.783 \\
\hline$<24.00$ & $368(37.9)$ & $39(28.3)$ & $329(39.5)$ & & $36(30.3)$ & $32(26.9)$ & \\
\hline $24.00-27.99$ & $388(40.0)$ & $62(44.9)$ & $326(39.1)$ & & $50(42.0)$ & $55(46.2)$ & \\
\hline$\geq 28.00$ & $215(22.1)$ & $37(26.8)$ & $178(21.4)$ & & $33(27.7)$ & $32(26.9)$ & \\
\hline Central obesity & & & & $<0.001$ & & & 0.697 \\
\hline Yes & $625(64.4)$ & $66(47.8)$ & $559(67.1)$ & & $60(50.4)$ & $57(47.9)$ & \\
\hline No & $346(35.6)$ & $72(52.2)$ & $274(32.9)$ & & $59(49.6)$ & $62(52.1)$ & \\
\hline \multicolumn{8}{|l|}{ Mean \pm SD } \\
\hline Age & & $52.3 \pm 11.3$ & $57.5 \pm 11.3$ & $<0.001$ & $53.2 \pm 11.9$ & $51.9 \pm 11.9$ & 0.401 \\
\hline Duration of T2DM & & $5.4 \pm 6.0$ & $7.5 \pm 6.6$ & 0.001 & $5.8 \pm 6.3$ & $5.9 \pm 6.1$ & 0.937 \\
\hline BMI & & $25.8 \pm 3.7$ & $25.3 \pm 4.1$ & 0.143 & $25.8 \pm 3.9$ & $25.9 \pm 3.9$ & 0.861 \\
\hline WC & & $90.9 \pm 8.4$ & $88.3 \pm 8.8$ & 0.001 & $90.9 \pm 8.7$ & $90.7 \pm 8.4$ & 0.825 \\
\hline
\end{tabular}

day and LEAD risk (after adjusting for age, gender, region, occupation, smoking status, BMI, WC, and T2DM duration, $P=0.005)$. In addition, compared with patients who did not consume alcohol, patients who had consumed alcohol for $>20$ years had a higher risk of LEAD after adjusting for various factors (OR: 3.48, 95\% CI: 1.0911.15), and a dose-response relationship between the number of years of alcohol use and the risk of LEAD was also observed $(P=0.019)$ (Table 2$)$.

When alcohol consumption was analysed as a continuous outcome, models A to $\mathrm{C}$ showed that increased alcohol consumption was associated with an increased risk of LEAD (all $P<0.05$, Table 2). However, the association between continuous years of alcohol consumption and LEAD was not significant after adjusting for various factors (Table 2). Furthermore, we utilized model D (adjusting for age, gender, region, occupation, smoking status, BMI, WC, T2DM duration, systolic blood pressure, cholesterol, and prevalent cardiovascular disease) to analyse participants with cholesterol, blood pressure, and prevalent cardiovascular disease data $(n=185)$ and obtained similar results (Table S1 available online at https://doi.org/10.1155/2017/8756978).

The gender imbalance between alcohol consumers and nonconsumers is striking. We performed an analysis on male patients only (the number of alcohol-consuming women was too low for a separate analysis of female patients). Compared with male patients who did not consume alcohol, male patients who consumed alcohol had a higher risk of LEAD (OR: 3.17, 95\% CI: 1.25-8.09) after adjusting for age, region, occupation, smoking status, BMI, WC, and T2DM duration. Regarding the risk of LEAD after adjusting for alcohol use $\leq 8 \mathrm{U} /$ day and $>8 \mathrm{U} /$ day, the ORs were 2.43 (95\% CI: $0.89-$ 6.68, $P>0.05$ ) and 7.03 (95\% CI: 1.91-25.84, $P<0.05$ ), respectively. We also observed a dose-response relationship between the units of alcohol consumed per day and LEAD risk (after adjusting for age, region, occupation, smoking status, BMI, WC, and T2DM duration, $P=0.003$ ). In addition, compared with male patients who did not consume alcohol, 
TABLE 2: OR (95\% CI) of LEAD in participants according to alcohol use.

\begin{tabular}{|c|c|c|c|c|}
\hline & $n(\%)$ & $\begin{array}{c}\text { Model A } \\
\text { OR (95\% CI) }\end{array}$ & $\begin{array}{c}\text { Model B } \\
\text { OR (95\% CI) }\end{array}$ & $\begin{array}{c}\text { Model C } \\
\text { OR }(95 \% \text { CI })\end{array}$ \\
\hline \multicolumn{5}{|l|}{ Alcohol use } \\
\hline No (reference) & $9(7.6)$ & 1 & 1 & 1 \\
\hline Yes & $21(17.6)$ & $2.62(1.15-5.99)$ & $2.61(1.09-6.23)$ & $2.75(1.11-6.80)$ \\
\hline$P$ & & 0.022 & 0.031 & 0.028 \\
\hline \multicolumn{5}{|l|}{ Alcohol consumption } \\
\hline No (reference) & $9(7.6)$ & 1 & 1 & 1 \\
\hline$\leq 8 \mathrm{U} /$ day & $14(15.7)$ & $2.28(0.94-5.54)$ & $1.95(0.76-5.02)$ & $2.07(0.78-5.54)$ \\
\hline$>8 \mathrm{U} /$ day & $7(23.3)$ & $3.72(1.26-11.01)$ & $6.33(1.89-21.15)$ & $6.35(1.78-22.65)$ \\
\hline$P$ for trend & & 0.012 & 0.004 & 0.005 \\
\hline \multicolumn{5}{|l|}{ Alcohol use duration } \\
\hline No (reference) & $9(7.6)$ & 1 & 1 & 1 \\
\hline$\leq 20$ years & $8(15.1)$ & $2.17(0.79-5.99)$ & $2.25(0.86-5.90)$ & $2.41(0.88-6.60)$ \\
\hline$>20$ years & $13(19.7)$ & $3.00(1.21-7.45)$ & $3.40(1.13-10.23)$ & $3.48(1.09-11.15)$ \\
\hline$P$ for trend & & 0.017 & 0.015 & 0.019 \\
\hline \multicolumn{5}{|l|}{ Continuous } \\
\hline No (reference) & & 1 & 1 & 1 \\
\hline Alcohol consumption (U) & & $1.06(1.00-1.12)$ & $1.10(1.03-1.18)$ & $1.11(1.04-1.19)$ \\
\hline$P$ & & 0.048 & 0.004 & 0.003 \\
\hline No (reference) & & 1 & 1 & 1 \\
\hline Alcohol use duration (years) & & $1.03(1.01-1.06)$ & $1.02(0.99-1.04)$ & $1.02(0.99-1.05)$ \\
\hline$P$ & & 0.013 & 0.054 & 0.055 \\
\hline
\end{tabular}

Model A: crude model; model B: adjusted for age, gender, region, and occupation; model C: adjusted for age, gender, region, occupation, smoking status, BMI, $\mathrm{WC}$, and T2DM duration.

male patients who had consumed alcohol for $>20$ years had a higher risk of LEAD after adjusting for various factors (OR: 2.82, 95\% CI: 1.07-7.91), and a dose-response relationship between the number of years of alcohol use and the risk of LEAD was also observed $(P=0.039)$ (Table 3$)$.

When alcohol consumption was analysed as a continuous outcome, models A to $\mathrm{C}$ showed that increased alcohol consumption was associated with an increased risk of LEAD (all $P<0.05$, Table 3 ). In addition, the association between continuous years of alcohol consumption and LEAD was not significant after adjusting for various factors (Table 3). Furthermore, we utilized model D (adjusting for age, origin, occupation, smoking status, BMI, WC, T2DM duration, systolic blood pressure, cholesterol, and prevalent cardiovascular disease) to analyse male participants with cholesterol, blood pressure, and prevalent cardiovascular disease data $(n=182)$ and obtained similar results (Table S2).

\section{Discussion}

In this study, we observed a significant association between alcohol consumption and LEAD in patients with T2DM. We used a standard and universal measure of alcohol consumption; in China, individuals usually drink white spirits distilled from sorghum or maize or beer, and "liang" (50 g) is usually used as the measurement for the amount of alcohol consumed [20]. These differences from Western countries make comparisons between nations difficult; however, we used the standard and universal measure $\mathrm{U}$, that is, $10 \mathrm{ml}$ or $8 \mathrm{~g}$ of ethanol, to solve this problem. We also used PSM to comprehensively control and adjust for a wide range of potential confounders and to improve the comparability between the two groups.

As shown in a study by Mukamal et al. [10], older adults in Pennsylvania who consumed 1-13 drinks per week had a lower risk (OR: 0.56, 95\% CI: 0.33-0.95) of hospitalization for LEAD; however, this reduced risk became insignificant when $>13$ drinks were consumed per week. This study used drinks per week as a measurement of alcohol consumption and did not present information about the quantity of alcohol consumed or the years of alcohol consumption. Vliegenthart et al. [11] observed an inverse relationship between alcohol consumption and PAD in nonsmoking women, but not in nonsmoking men; this difference may be related to the propensity of males to drink beer, wine, and liquor, whereas females predominantly drink wine and types of fortified wine. In addition, Xie et al. [21] observed an association between heavy drinking (>60 g/ day) and a higher risk (OR: 2.878, 95\% CI: 1.215-4.018) of a low ABI in Chinese men; this result is consistent with the findings of our study. Furthermore, heavy drinking has adverse effects on blood pressure and serum triglyceride levels [22], both of which may lead to LEAD.

This study had several limitations. As the information on alcohol consumption was based on recall, recall bias could not be completely excluded; however, the information was 
TABLE 3: OR (95\% CI) of LEAD in male participants according to alcohol use.

\begin{tabular}{|c|c|c|c|c|}
\hline & $n(\%)$ & $\begin{array}{c}\text { Model A } \\
\text { OR (95\% CI) }\end{array}$ & $\begin{array}{c}\text { Model B } \\
\text { OR (95\% CI) }\end{array}$ & $\begin{array}{c}\text { Model C } \\
\text { OR }(95 \% \text { CI })\end{array}$ \\
\hline \multicolumn{5}{|l|}{ Alcohol use } \\
\hline None (reference) & $8(6.8)$ & 1 & 1 & 1 \\
\hline Yes & $21(17.9)$ & $2.98(1.26-7.04)$ & $3.10(1.25-7.67)$ & $3.17(1.25-8.09)$ \\
\hline$P$ & & 0.013 & 0.015 & 0.016 \\
\hline \multicolumn{5}{|l|}{ Alcohol consumption } \\
\hline None (reference) & $8(6.8)$ & 1 & 1 & 1 \\
\hline$\leq 8 \mathrm{U} /$ day & $14(16.1)$ & $2.61(1.04-6.54)$ & $2.36(0.88-6.26)$ & $2.43(0.89-6.68)$ \\
\hline$>8 \mathrm{U} /$ day & $7(23.3)$ & $4.15(1.37-12.58)$ & $7.32(2.10-25.42)$ & $7.03(1.91-25.84)$ \\
\hline$P$ for trend & & 0.007 & 0.002 & 0.003 \\
\hline \multicolumn{5}{|l|}{ Alcohol use duration } \\
\hline None (reference) & $8(6.8)$ & 1 & 1 & 1 \\
\hline$\leq 20$ years & $8(15.1)$ & $2.42(0.86-6.85)$ & $3.88(1.25-12.07)$ & $3.93(1.19-12.96)$ \\
\hline$>20$ years & $13(20.3)$ & $3.47(1.36-8.90)$ & $2.74(1.01-7.39)$ & $2.82(1.01-7.91)$ \\
\hline$P$ for trend & & 0.009 & 0.042 & 0.039 \\
\hline \multicolumn{5}{|l|}{ Continuous } \\
\hline None (reference) & & 1 & 1 & 1 \\
\hline Alcohol consumption (U) & & $1.06(1.00-1.13)$ & $1.11(1.04-1.18)$ & $1.11(1.04-1.19)$ \\
\hline$P$ & & 0.036 & 0.003 & 0.003 \\
\hline None (reference) & & 1 & 1 & 1 \\
\hline Alcohol use duration (years) & & $1.04(1.01-1.07)$ & $1.03(1.00-1.05)$ & $1.03(1.00-1.06)$ \\
\hline$P$ & & 0.004 & 0.067 & 0.063 \\
\hline
\end{tabular}

Model A: crude model; model B: adjusted for age, region, and occupation; model C: adjusted for age, region, occupation, smoking status, BMI, WC, and T2DM duration.

confirmed by patients and their relatives to ensure accuracy. Second, our sample may not be completely representative of patients with T2DM in China because our hospital is one of the best hospitals in Zibo, and the inpatients here have higher proportions of diabetic complications. However, the representativeness of our sample should not substantially affect the internal validity of this study. Third, the cholesterol, blood pressure, and prevalent cardiovascular disease data were missing for 229 participants, and thus, we did not include these three variables as confounders for PSM. Furthermore, we did not collect information about physical activity or homocysteine levels, which are also risk factors for LEAD [23]. However, we utilized model D (adjusting for age, gender, region, occupation, smoking status, BMI, WC, T2DM duration, systolic blood pressure, cholesterol, and prevalent cardiovascular disease) in participants with available cholesterol, blood pressure, and prevalent cardiovascular disease data $(n=185)$ and obtained similar results, as the effect of alcohol consumption on the LEAD risk was still significant after adjusting for these factors. Finally, we could not examine the hazard ratio of alcohol consumption with respect to LEAD because detailed information regarding the onset time of LEAD was not available.

In summary, our study observed a dose-response relationship between alcohol consumption and LEAD among inpatients with T2DM. We used a standard and universal measurement of alcohol consumption and increased the comparability of the two groups using the PSM method. Alcohol consumption may be a risk factor for LEAD in patients with T2DM; however, further cohort studies should be conducted to verify the causal relationship. Based on our findings, patients with T2DM should be advised to stop drinking, or at least to avoid heavy drinking, to prevent the onset of LEAD.

\section{Abbreviations}

ABI: Ankle-brachial index

BMI: $\quad$ Body mass index

95\% CI: $95 \%$ confidence interval

DM: Diabetes mellitus

LEAD: Lower extremity arterial disease

OR: Odds ratio

PAD: Peripheral artery disease

PSM: $\quad$ Propensity score matching

T2DM: Type 2 diabetes mellitus

TBI: Toe-brachial index

WC: Waist circumference.

\section{Conflicts of Interest}

The authors declare that they have no conflicts of interest.

\section{Authors' Contributions}

Dr. Shanshan Yang and Professor Zhengguo Yang conceived and designed the study; Shuang Wang, Bo Yang, Jinliang 
Zheng, and Yuping Cai performed the experiments; Shanshan Yang and Shuang Wang analysed the data; and Shanshan Yang, Shuang Wang, and Zhengguo Yang contributed materials/analysis tools and wrote the paper. Shanshan Yang and Shuang Wang contributed equally to this work. This manuscript has been read and approved by all the authors, the requirements for authorship have been met, and each author believes that the manuscript represents honest work.

\section{Acknowledgments}

The authors would like to thank Lei Xu, Qian Li, Wei Jia, Xinli Cai, Lihui Liu, Ying Zhang, Jinjuan Zhao, and Xinai Yan from the 148th PLA Hospital for assisting with the research described in this study.

\section{References}

[1] R. E. Hoyt, "Peripheral arterial disease in people with diabetes: response to consensus statement," Diabetes Care, vol. 27, no. 8, p. 2095, 2004.

[2] J. Malmstedt, L. Karvestedt, J. Swedenborg, and K. Brismar, "The receptor for advanced glycation end products and risk of peripheral arterial disease, amputation or death in type 2 diabetes: a population-based cohort study," Cardiovascular Diabetology, vol. 14, no. 1, p. 93, 2015.

[3] J. R. Brownrigg, N. C. Schaper, and R. J. Hinchliffe, "Diagnosis and assessment of peripheral arterial disease in the diabetic foot," Diabetic Medicine, vol. 32, no. 6, pp. 738-747, 2015.

[4] J. Muasher, "Lower extremity arterial disease," Journal of Vascular and Interventional Radiology, vol. 23, no. 7, pp. 186-205, 2012.

[5] C. D. Society, "Guidelines for prevention and treatment of type 2 diabetes mellitus in China," Chinese Jounal of Diabetes, vol. 22, no. 8, pp. 2-42, 2014.

[6] C. D. Society, "Guidelines for prevention and treatment of type 2 diabetes mellitus in China," Chinese Jounal of Diabetes, vol. 20, no. 1, pp. S1-S37, 2012.

[7] M. E. Levin, "Preventing amputation in the patient with diabetes," Diabetes Care, vol. 18, no. 10, pp. 1383-1394, 1995.

[8] N. G. Mahon, P. O. Rahallaigh, J. B. O'Sullivan, M. B. Codd, H. A. McCann, and D. D. Sugrue, "Hospital cost of acute myocardial infarction in the thrombolytic era," Irish Medical Journal, vol. 93, no. 4, p. 122, 2000.

[9] I. Wakabayashi and Y. Sotoda, "Alcohol drinking and peripheral arterial disease of lower extremity," Nihon Arukōru Yakubutsu Igakkai Zasshi, vol. 49, no. 1, pp. 13-27, 2014.

[10] K. Mukamal, M. Kennedy, M. Cushman et al., "Alcohol consumption and lower extremity arterial disease among older adults the cardiovascular health study," American Journal of Epidemiology, vol. 167, no. 1, pp. 34-41, 2008.

[11] R. Vliegenthart, J. M. Geleijnse, A. Hofman et al., "Alcohol consumption and risk of peripheral arterial disease: the Rotterdam Study," American Journal of Epidemiology, vol. 155, no. 4, pp. 332-338, 2002.

[12] E. Sacanella and R. Estruch, "The effect of alcohol consumption on endothelial adhesion molecule expression," Addiction Biology, vol. 8, no. 4, pp. 371-378, 2004.

[13] D. Power, "Standards of medical care in diabetes," Diabetes Care, vol. 29, no. 2, pp. S4-S36, 2006.
[14] M. Ruizcanela and M. A. Martínezgonzález, "Lifestyle and dietary risk factors for peripheral artery disease," Circulation Journal, vol. 78, no. 3, pp. 553-559, 2014.

[15] I. Wakabayashi, "Comparison of the relationships of alcohol intake with atherosclerotic risk factors in men with and without diabetes mellitus," Alcohol and Alcoholism (Oxford), vol. 46, no. 3, pp. 301-307, 2011.

[16] J. H. O’Keefe, S. K. Bhatti, A. Bajwa, J. J. Dinicolantonio, and C. J. Lavie, "Alcohol and cardiovascular health: the dose makes the poison...or the remedy," Mayo Clinic Proceedings, vol. 89, no. 3, pp. 382-393, 2014.

[17] J. Xiao, X. Xing, J. Lu et al., "Prevalence and associated factors of microalbuminuria in Chinese individuals without diabetes: cross-sectional study," BMJ Open, vol. 3, no. 11, article e003325, 2013.

[18] F. Thoemmes, "Propensity score matching in SPSS," ArXiv Preprint, vol. 6385, p. 1201, 2012.

[19] P. C. Austin, "Optimal caliper widths for propensity-score matching when estimating differences in means and differences in proportions in observational studies," Pharmaceutical Statistics, vol. 10, no. 2, pp. 150-161, 2011.

[20] G. S. Ma, D. H. Zhu, X. Q. Hu, D. C. Luan, L. Z. Kong, and X. G. Yang, "The drinking practice of people in China," Acta Nutrimenta Sinica, vol. 27, no. 5, pp. 362-365, 2005.

[21] X. Xie, Y. T. Ma, Y. N. Yang et al., "Alcohol consumption and ankle-to-brachial index: results from the cardiovascular risk survey," PLoS One, vol. 5, no. 12, article e15181, 2010.

[22] I. Wakabayashi, R. Kobaba-Wakabayashi, and H. Masuda, "Relation of drinking alcohol to atherosclerotic risk in type 2 diabetes," Diabetes Care, vol. 25, no. 7, pp. 1223-1228, 2002.

[23] M. D. Gerhard-Herman, H. L. Gornik, C. Barrett et al., "2016 AHA/ACC guideline on the management of patients with lower extremity peripheral artery disease: a report of the American College of Cardiology/American Heart Association task force on clinical practice guidelines," Journal of the American College of Cardiology, vol. 69, no. 11, p. 1465, 2017. 


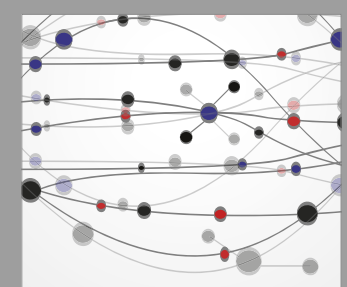

The Scientific World Journal
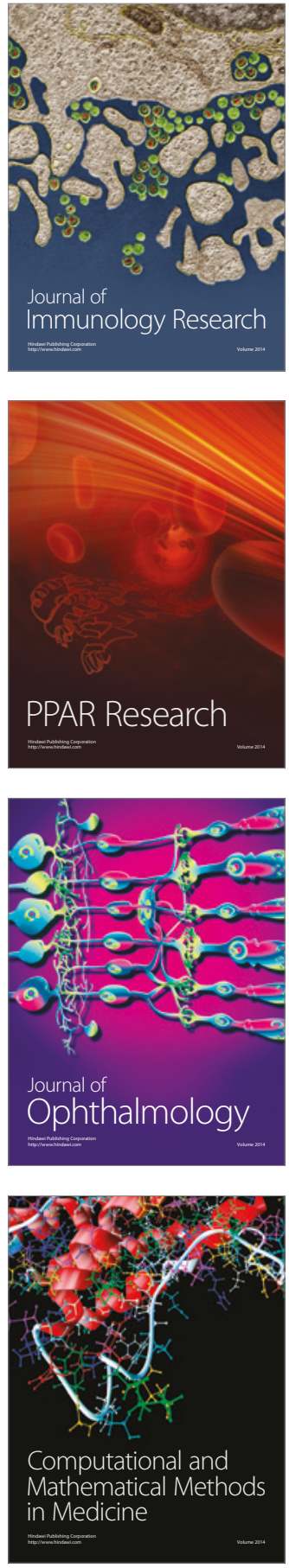

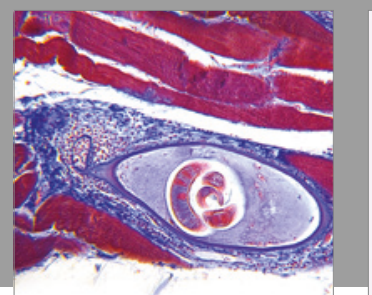

Gastroenterology Research and Practice
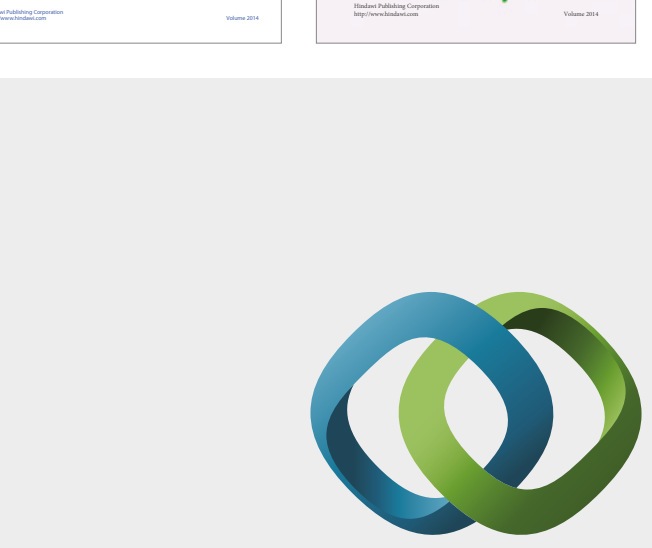

\section{Hindawi}

Submit your manuscripts at

https://www.hindawi.com
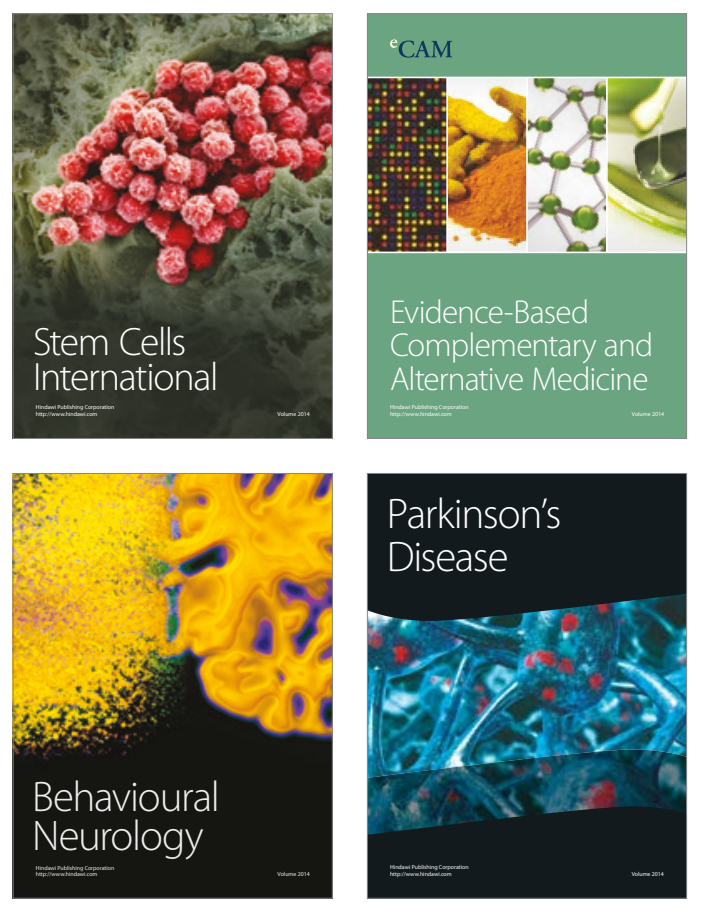
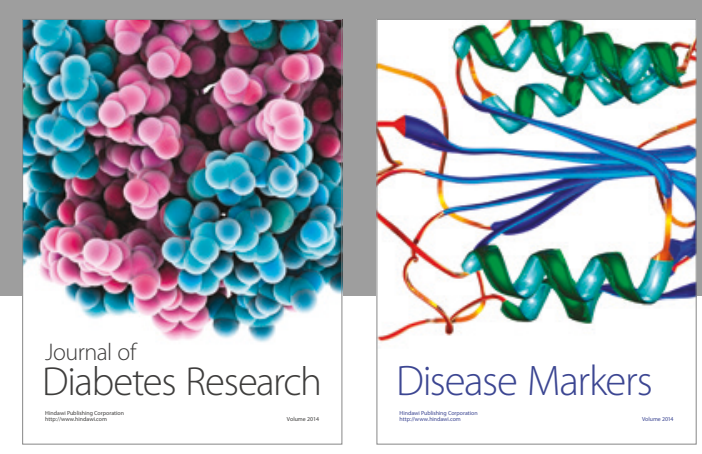

Disease Markers
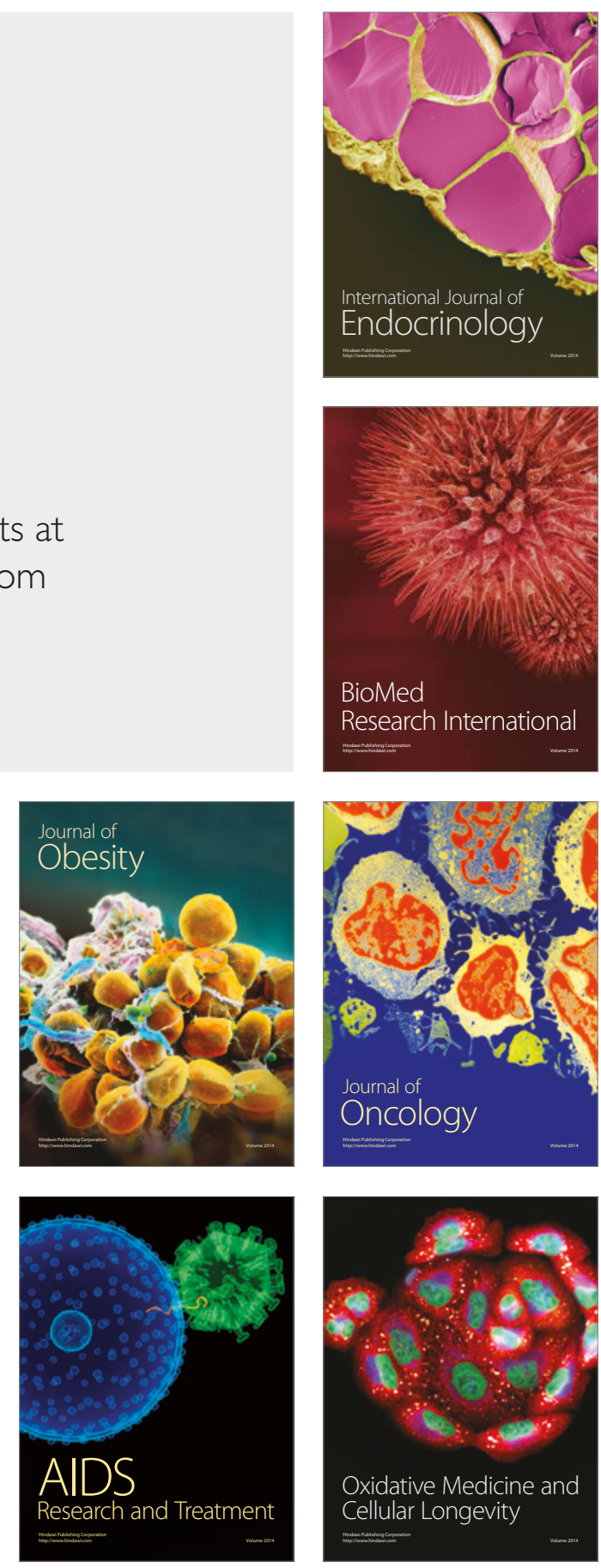